\title{
Autophagy is central to joint destruction in arthritis
}

A new study provides evidence of an important role of autophagy in joint destruction in rheumatoid arthritis (RA). The findings demonstrate that autophagy regulates osteoclast differentiation and bone resorption in a TNF-dependent manner, and suggest that interfering with this process could prevent structural damage in arthritis.

"Several proinflammatory cytokines, including IL-1 and IL-6, as well as TLR ligands, have been shown to stimulate autophagy in other cell types," explain Neng-Yu Lin and Jörg Distler, two of the study's authors. "Considering the role of those pathways in RA and the role of autophagy in tissue homeostasis, we hypothesized that autophagy may play a role in the pathogenesis of RA."

The researchers first showed that autophagy is activated in osteoclasts

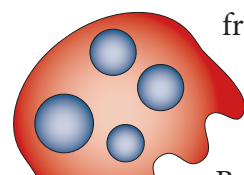
from patients with RA, as demonstrated by increased expression of two important regulators of autophagy, Beclin1 and Atg7, in these cells in comparison with cells from patients with osteoarthritis. The authors hypothesized that TNF, a key factor in inflammatory bone resorption in arthritis, might be implicated in this process. In keeping with this theory, expression of both Beclin1 and Atg7 was increased in mouse osteoclasts both in vitro, upon stimulation with TNF (and particularly in conditions with low concentrations of RANKL), and in vivo, in TNF transgenic mice.

Closer examination of autophagosomes and organelle degradation in wildtype and Atg7-deficient osteoclasts by electron microscopy suggested that impaired autophagy might interfere with osteoclastogenesis in these cells. To test this hypothesis, the researchers modulated autophagy in vitro and in vivo by use of a small-molecule inhibitor of autophagy, LysMCre-mediated knockout of Atg7, and lentiviral overexpression of Beclin1. "Activation of autophagy by overexpression of Beclin1 induced osteoclastogenesis and enhanced the resorptive capacity of cultured osteoclasts, whereas pharmacologic or genetic inactivation of autophagy prevented osteoclast differentiation," report Lin and Distler. Further assays revealed that the reduced bone-resorbing capacity of mouse bone marrow cells following pharmacologic or genetic inhibition of autophagy was attributable to both decreased numbers of osteoclasts and impaired osteoclast function.

Turning to the effects of autophagy in experimental arthritis, the researchers analyzed the extent of bone and cartilage destruction in TNF transgenic mice after adoptive transfer with myeloidspecific Atg7-deficient bone marrow cells, which selectively targeted autophagy in monocytes. Recipient mice showed reduced numbers of osteoclasts compared with control mice, and were protected from TNF-induced bone erosion, proteoglycan loss and chondrocyte death; notably, clinical signs of inflammation were not affected. "Inhibition of autophagy may thus

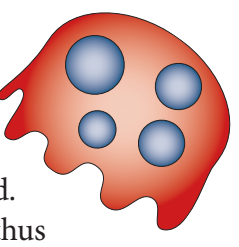
prevent osteoclast differentiation and activation, and inhibit bone erosions in RA," the authors conclude. Future studies will aim to further elucidate the molecular mechanisms by which autophagy regulates osteoclastogenesis.

Sarah Onuora

Original article Lin, N.-Y. et al. Autophagy regulates TNFamediated joint destruction in experimental arthritis. Ann. Rheum. Dis. doi:10.1136/annrheumdis-2012-201671 